\title{
Research Paper \\ Comparing Family-related Factors, Nutritional-behavioral Habits, and Lifestyle Between Obese and Non-obese Children
}

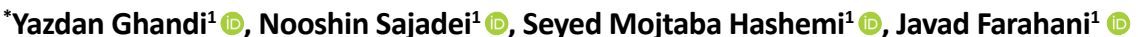

1. Department of Pediatrics, School of Medicine, Arak University of Medical Sciences, Arak, Iran.

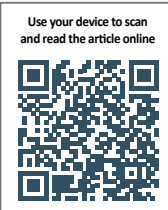

CIttation: Ghandi Y, Sajadei N, Hashemi SM, Farahani J. [Comparing Family-related Factors, Nutritional-behavioral Habits, and Lifestyle Between Obese and Non-obese Children (Persian)]. Journal of Arak University of Medical Sciences (JAMS). 2021; 24(2):278-291. https://doi.org/10.32598/JAMS.24.2.6229.1

d $)$ inttps://doi.org/10.32598/JAMS.24.2.6229.1

Key words:

Child, Lifestyle, Nutrition, Obesity

\section{ABSTRACT}

Article Info:

Received: 23 Jun 2020

Accepted: 18 May 2021

Available Online: 01 Jun 2021

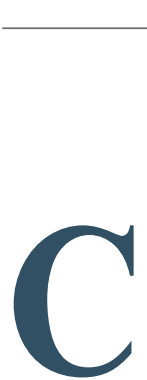

\section{Extended Abstract}

\section{Introduction}

hildhood obesity is among the major health problems worldwide [1]. Obesity and overweight in children are also risk factors for adult obesity [5]. Obesity is increasing among children and adolescents. In Iran, the prevalence of obesity is higher in adolescents [7]. Various risk factors for childhood obesity have been studied. Obesity is caused by several characteristics, including genetics, hormonal, metabolic, and behavioral factors [8]. The importance of nutritional behaviors and the problem of obesity derived from it has been raised as a serious problem for the present century [10].

\section{* Corresponding Author:}

\section{Yazdan Ghandi, PhD.}

Address: Department of Pediatrics, School of Medicine, Arak University of Medical Sciences, Arak, Iran.

Tel: +98 (912) 3833712

E-mail: drghandi1351@gmail.com 
Considering the significance of childhood obesity and its complications, as well as easier correction of related factors in this age group, the present study aimed to investigate the role of some factors related to family, nutrition, and lifestyle on childhood obesity.

\section{Materials and Methods}

This age-matched case study was performed on children referring to Amir Kabir Hospital in Arak City, Iran, after obtaining the code of ethics, in the spring and summer of 2019. The sample size in this study was estimated using the results of a similar study [14] and a 95\% Confidence Interval (CI) using STATA to equal 150 children per research group. To collect the necessary data, the weight and height of each child were initially measured. The weight was computed by a scale with an accuracy of $1 \mathrm{~kg}$ and in a situation where the child used a standard measuring tape with an accuracy of $1 \mathrm{~cm}$ to measure height. Then, Body Mass Index (BMI) and percentile were calculated for each examined child.

Data collection was performed, using a three-part checklist, including information about birth, the type of delivery, the presence of consanguineous marriage, birth weight, the number of children in the family, nutritional data, and lifestyle information. The obtained data were analyzed using STATS at a $95 \%$ CI.

\section{Results}

Table 1 indicates that childhood obesity was significantly related to the father's age, parents' BMI, and the number of children in the family $(\mathrm{P}<0.05)$. Moreover, according

Table 1. Relationship between childhood obesity and family-related variables

\begin{tabular}{|c|c|c|c|c|c|c|}
\hline \multirow{2}{*}{\multicolumn{2}{|c|}{ Characteristic }} & \multicolumn{2}{|c|}{ No. (\%) } & \multirow{2}{*}{$\mathbf{P}$} & \multirow{2}{*}{ OR } & \multirow{2}{*}{$95 \% \mathrm{Cl}$} \\
\hline & & Non-Obese Group & Obese Group & & & \\
\hline \multirow{3}{*}{ Gender } & Female & $57(38)$ & $66(44)$ & & 1 & \\
\hline & & & & 0.291 & & \\
\hline & Male & $93(62)$ & $84(56)$ & & 0.78 & $0.47-1.26$ \\
\hline \multirow{3}{*}{ Father's age, y } & $<25$ & $4(27)$ & $15(10)$ & & 1 & \\
\hline & $25-35$ & $88(58.7)$ & $81(54)$ & 0.033 & 0.24 & $0.05-0.81$ \\
\hline & $>35$ & $58(36.8)$ & $54(36)$ & & 0.24 & $0.05-0.85$ \\
\hline \multirow{3}{*}{ Mother's age, y } & $<25$ & $7(4.7)$ & $15(10)$ & & 1 & $0.15-1.25$ \\
\hline & $25-35$ & 101(67.3) & $99(66)$ & 0.184 & 0.45 & $0.12-1.19$ \\
\hline & $>35$ & $42(28)$ & $36(24)$ & & 0.4 & \\
\hline \multirow{3}{*}{ Father's BMI, kg/m² } & Healthy & 119(79.3) & $75(50)$ & & 1 & \\
\hline & Overweight & $27(18)$ & $66(44)$ & 0.000 & 3.87 & $2.2-6.87$ \\
\hline & Obese & $4(2.7)$ & $9(6)$ & & 3.57 & $1.1-16.33$ \\
\hline \multirow{3}{*}{ Mother's BMI, kg/m² } & Healthy & 106(70.7) & $57(38)$ & & 1 & \\
\hline & Overweight & $41(27.3)$ & $84(56)$ & 0.000 & 3.81 & $2.26-6.43$ \\
\hline & Obese & $3(2)$ & $9(6)$ & & 5.57 & $1.31-32.9$ \\
\hline \multirow{3}{*}{$\begin{array}{l}\text { The number of } \\
\text { children }\end{array}$} & 1 & $21(14)$ & $9(6)$ & & 1 & \\
\hline & 2 & $87(58)$ & $81(54)$ & 0.009 & 2.17 & $0.88-5.69$ \\
\hline & $\geq 3$ & $42(28)$ & $60(40)$ & & 3.33 & $1.29-9.05$ \\
\hline
\end{tabular}

The significance level of the tests was calculated by Chi-squared test and the OR was calculated by logistic regression model with an EE of 0.05 . 
Table 2. Relationship between childhood obesity and nutrition-related variables

\begin{tabular}{|c|c|c|c|c|c|c|}
\hline \multirow{2}{*}{ Characteristic } & & \multicolumn{2}{|c|}{ No. (\%) } & \multirow{2}{*}{$\mathbf{P}$} & \multirow{2}{*}{ OR } & \multirow{2}{*}{$95 \% \mathrm{Cl}$} \\
\hline & & Non-Obese Group & Obese Group & & & \\
\hline \multirow{3}{*}{ Consuming fruits \& vegetables } & Never & $3(2)$ & $12(8)$ & \multirow{3}{*}{0.0001} & 1 & \\
\hline & Low & $37(24.7)$ & $51(34)$ & & 0.34 & $0.05-0.41$ \\
\hline & High & $110(73.3)$ & $87(58)$ & & 0.19 & $0.03-0.76$ \\
\hline \multirow[b]{2}{*}{ Eating breakfast } & No & $13(8.7)$ & $27(18)$ & \multirow[b]{2}{*}{0.001} & 1 & \\
\hline & Yes & 137(91.3) & $123(82)$ & & 0.43 & $0.19-0.91$ \\
\hline \multirow{3}{*}{ Supplementation } & No & $116(77.3)$ & $84(56)$ & \multirow{3}{*}{0.0001} & 1 & \\
\hline & & & & & & \\
\hline & Yes & $34(22.7)$ & $66(44)$ & & 2.68 & $1.58-4.57$ \\
\hline \multirow{5}{*}{ Consuming snacks } & Non & $9(6)$ & $4(2.7)$ & \multirow{5}{*}{0.051} & 1 & \\
\hline & 1 & $6(4)$ & $13(8.7)$ & & 4.87 & $0.85-29.9$ \\
\hline & & & & & & \\
\hline & 2 & $78(52)$ & $91(60.6)$ & & 2.62 & $0.69-12.06$ \\
\hline & $\geq 3$ & $57(38)$ & $42(28)$ & & 1.65 & $0.42-7.83$ \\
\hline \multirow{3}{*}{ Eating out } & No & 73(51.3) & $57(38)$ & \multirow{3}{*}{0.062} & 1 & \\
\hline & & & & & & \\
\hline & yes & $77(48.7)$ & $93(63)$ & & 1.54 & $0.95-2.51$ \\
\hline \multirow{3}{*}{ Fast food consumption } & No & 129(86) & $91(60.7)$ & \multirow{3}{*}{0.0001} & 1 & \\
\hline & & & & & & \\
\hline & Yes & $21(14)$ & 59(39.3) & & 3.98 & 2.19-7.37 \\
\hline
\end{tabular}

The significance level of the tests was calculated by Chi-squared test and the OR was calculated by logistic regression model with an EE of 0.05.

to Table 2, there was a significant relationship between obesity and the consumption of fruits and vegetables, fast food, and breakfast, dietary supplementation $(\mathrm{P}<0.05)$. The Odds Ratio (OR) data revealed that the odds of obesity in children who consume fewer fruits and vegetables and those who consume more of these edibles was higher than those who did not consume at all, respectively was equal to 0.3 (95\%CI: 0.1-05.41) and 0.19 (95\%CI: 0.03-0.76).

Table 3 illustrates data concerning the relationship between obesity and variables related to lifestyle factors. There was a significant relationship between obesity in children and good chewing of food, participation in preparing food and table, and using food as a reward $(\mathrm{P}<0.05)$. Furthermore, the OR data suggested that the odds of becoming obese in children who played computer games for more than two hours (95\%CI: 1.08-12.37) was 3.5 times higher, compared to those who did not play computer games. The OR for good chewing of food, in comparison with children who did not chew food well, was equal to 0.38 (95\% CI: $0.2-0.69)$.

\section{Discussion and Conclusion}

The present study data indicated that the older the parents, the lower the child's obesity. Moreover, the odds of obesity increase in children with the enhanced number of children, as well as overweight and obesity of parents (i.e., a strong risk factor for obesity in children). According to some studies, factors related to parents, including maternal obesity, can also lead to childhood obesity $[15,16]$. The present study data revealed the consumption of fruits and vegetables and eating breakfast, as protective factors, and consumption of supplements and fast food, as risk factors for childhood obesity; these results were consistent with those of some studies [21-23].

Another eating habit of obese children is consuming multiple snacks, i.e., evaluated in this study. The OR results for 
Table 3. Relationship between childhood obesity and lifestyle-related variables

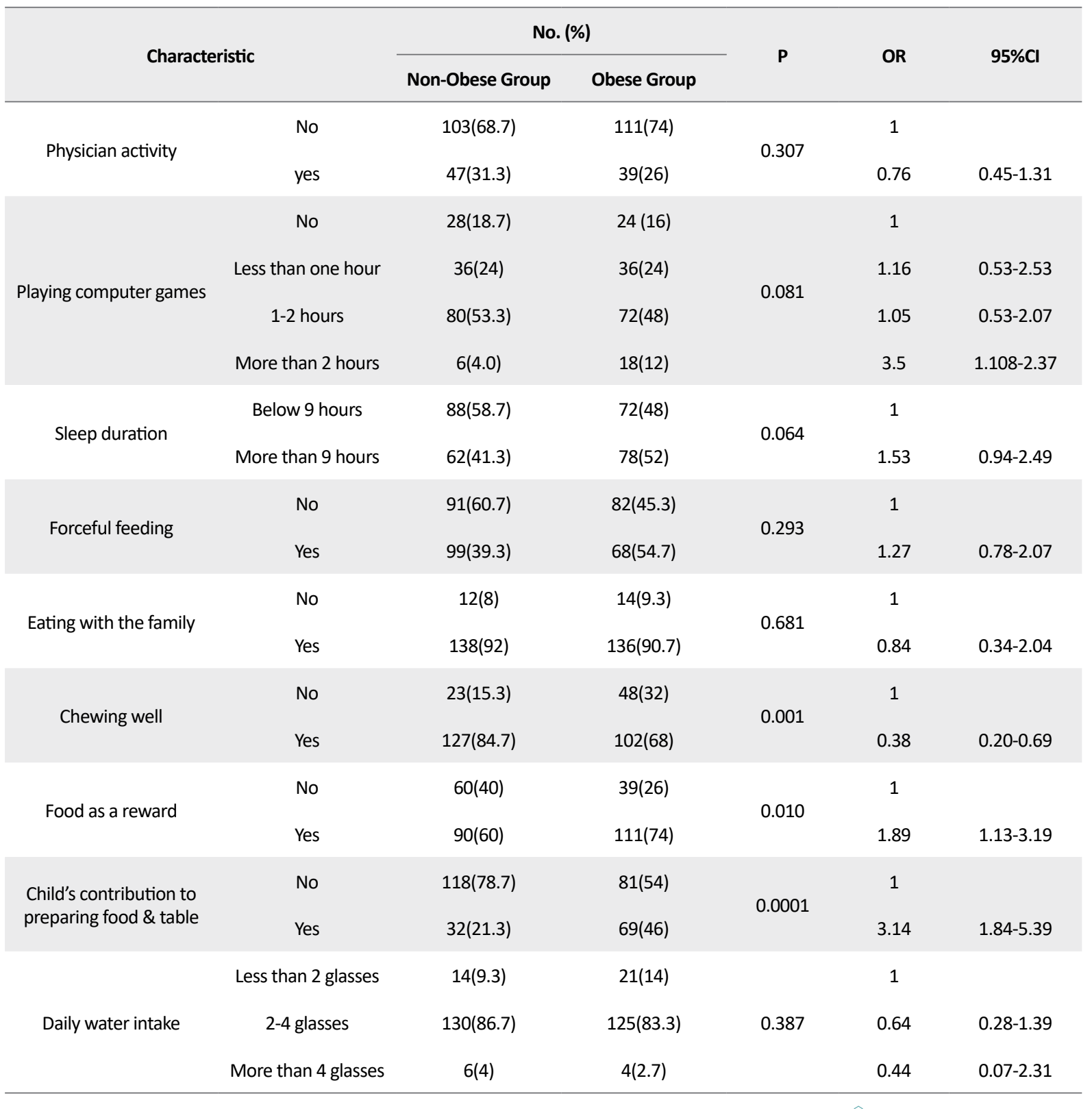

The significance level of the tests was calculated by Chi-squared test and the OR was calculated by logistic regression model with an EE of 0.05 .

these variables were not significant. Additionally, playing computer games for more than two hours, participating in the preparation of food and table, and using food as a reward was also mentioned as risk factors for childhood obesity. Some studies indicated that active video games prevent the occurrence of childhood obesity [32]. The present study findings found that with increasing sleep time, the child's chances of obesity increase. Studies have generally revealed that sleep disorders lead to weight gain for various reasons, including sedentary lifestyles, and that irregular sleep (either too much or too little) increases the odds of becoming obese [40].

Obesity is associated with some family-related characteristics, such as nutrition, playing computer games, and lifestyle factors. Due to the existence of various risk factors and adjustable protection, it is necessary to properly educate families and children to reduce obesity. 


\section{Ethical Considerations}

\section{Compliance with ethical guidelines}

This study was approved by the Ethics Committee of Arak University of Medical Sciences (Code: IR.ARAKMU. REC.1396.119). The research procedure was explained to the parents of the examined children and an informed written informed consent form was obtained from them.

Funding

This research did not receive any grant from funding agencies in the public, commercial, or non-profit sectors.

\section{Authors' contributions}

Conceptualization: Javad Farahani, Yazdan Ghandi; Data analysis: Javad Farahani; Investigation, writing - original draft, and writing - review \& editing, methodology: All authors.

\section{Conflicts of interest}

The authors declared no conflicts of interest.

\section{Acknowledgements}

The authors would like to thank the staff of the Amirkabir Hospital Clinical Research Development Center in Arak. 


\title{
مقايسه عادات تغذيهاى رفتارى و سبك زندگَى كودكان گاق و غير جاق: يك مطالعه مورد شاهدى
}

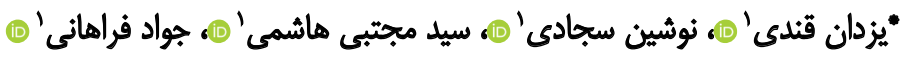

1. كروه اطفال، دانشكده يزشكى، دانشكاه علوم يزشكى اراك، اراك، ايران.

\begin{abstract}
حيكيد

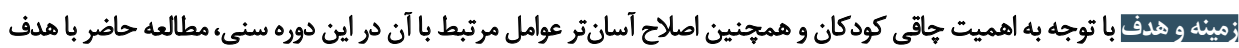

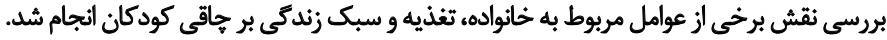

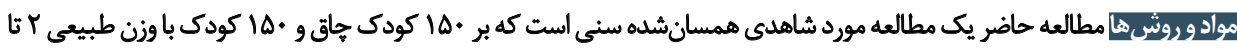

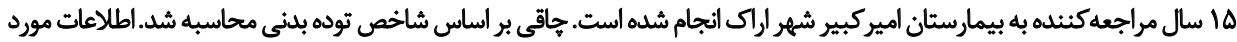

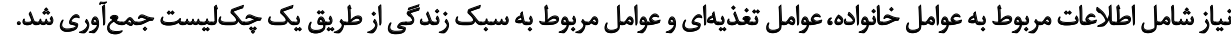

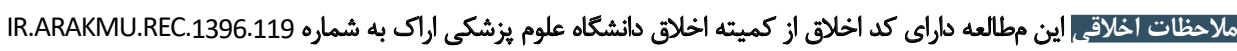

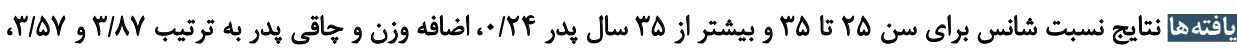

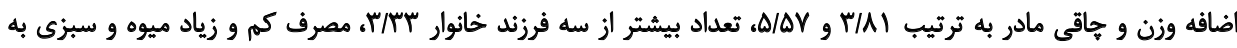

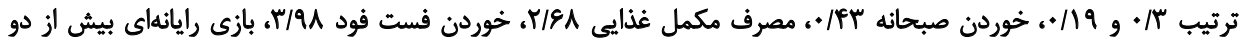

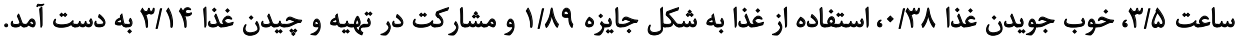

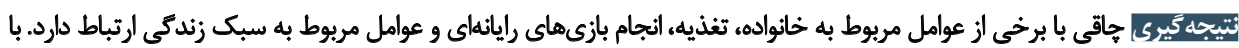

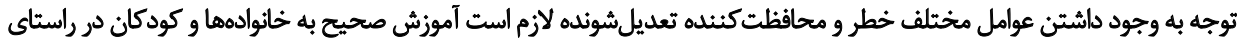

است. بسيارى از بيمارىهاى مزمن دوران بزركسالى ريشه در

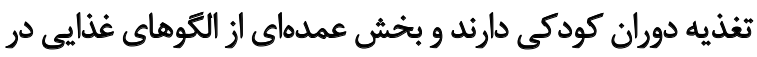

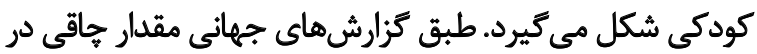

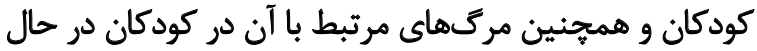

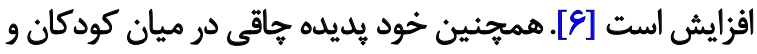

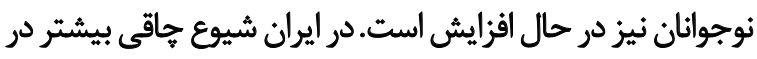

سنين نوجوانى ديده مي شود [V]

تاكنون عوامل خطر گوناتونى براى جاقى كودكان بررسى شده

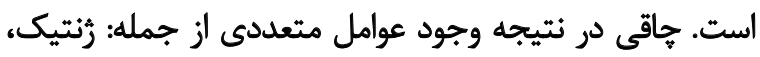

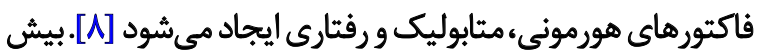

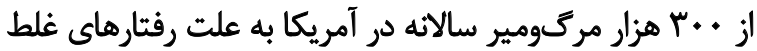

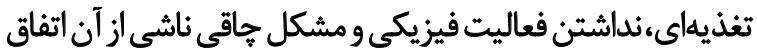

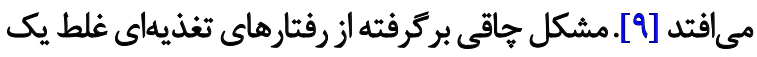

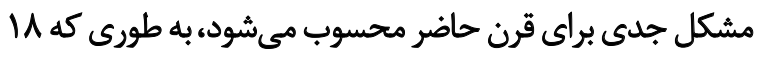

جاقي كودكان يكى از مشكلات عمده بهداشتى در سراسر دنيا

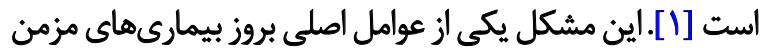

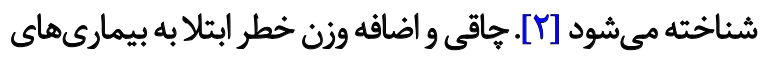

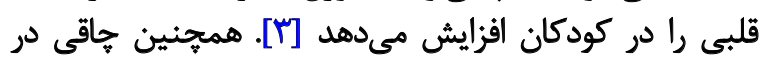

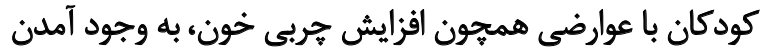

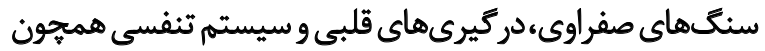

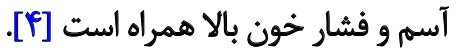

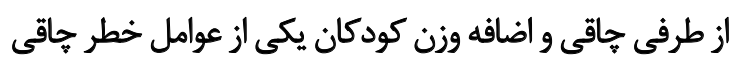

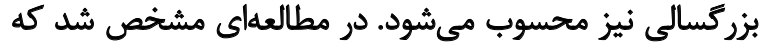

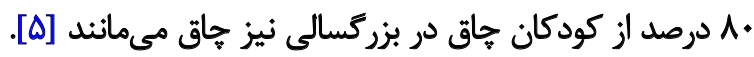

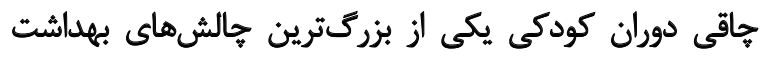

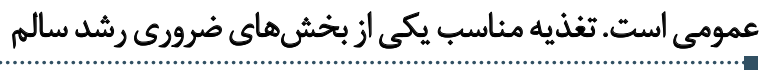

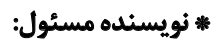

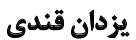
نشائى: اراك، دانشكاه علوم يزشكى اراك، دانشكده يزشكى، تروه اطفال.

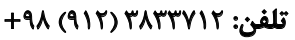
بست الكترونيكى: drghandi1351@gmail.com 
وزن، وجود بيمارىهايى همجيون بيمارىهاى روانى، سرطانها،

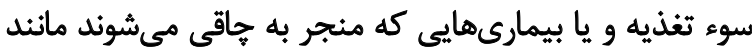

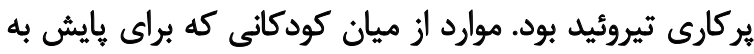

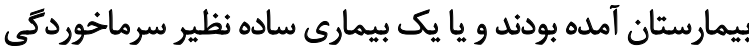

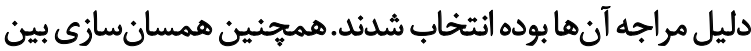
دو كروه از اين نظر نيز انجام كرديد.

براى جمعآورى اطلاعات ابتدا وزن و قد هر كودى اندازهكيرى

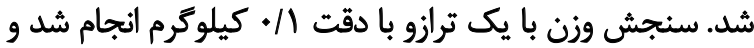

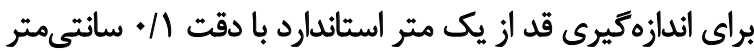

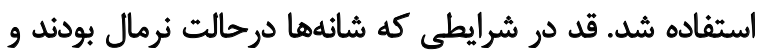

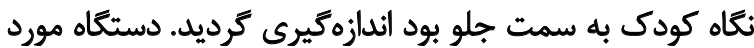

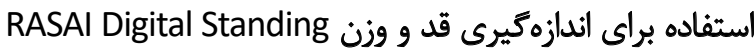

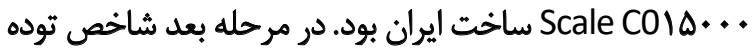

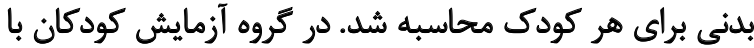

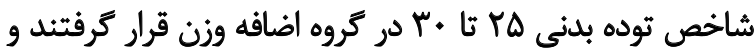

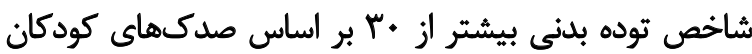

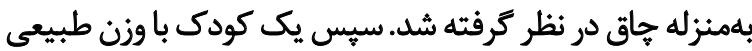

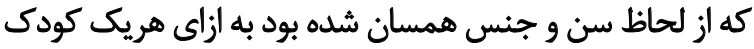

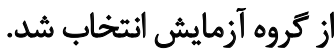

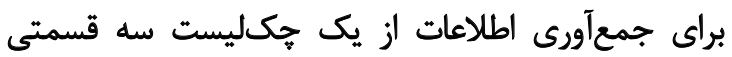

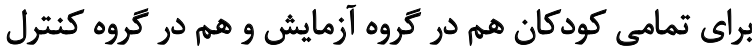

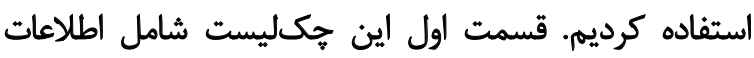

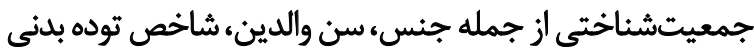

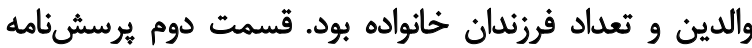

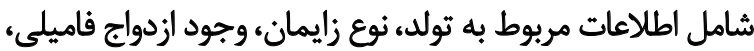

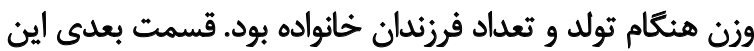

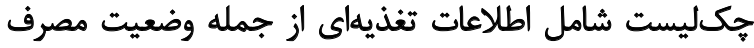

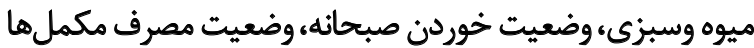

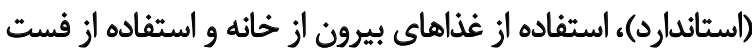

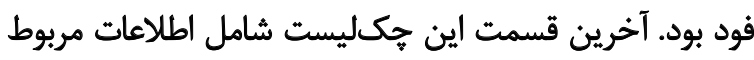

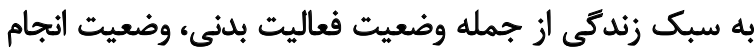

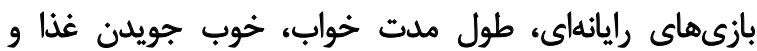

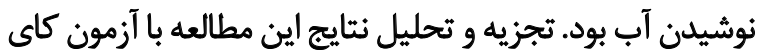

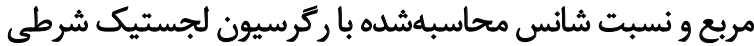

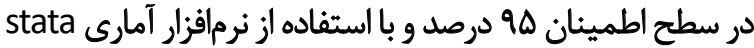

نسخه الانجام شد.

يافتهها

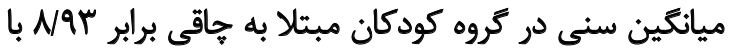

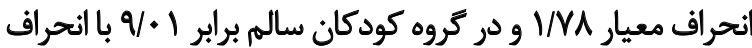

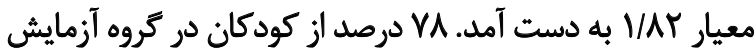

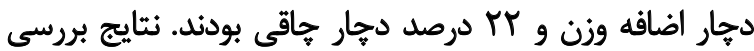

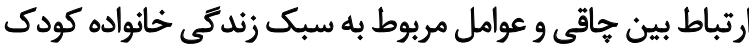

هدف از اهداف تصويبشده سازمان بهداشت جهانى با نام مردم

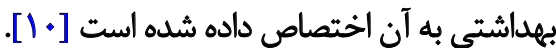

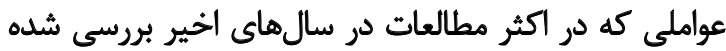

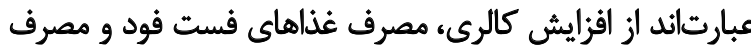

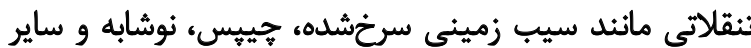

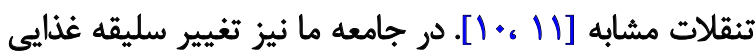

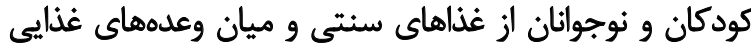

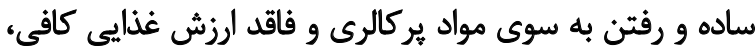

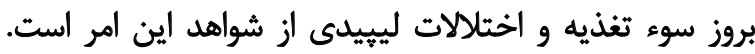

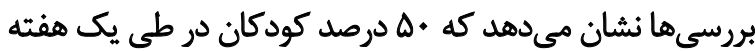

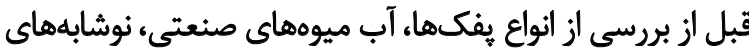

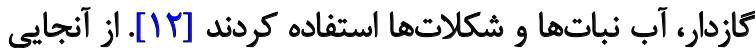

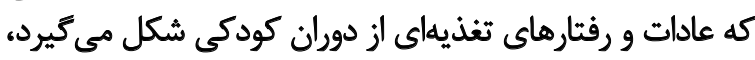

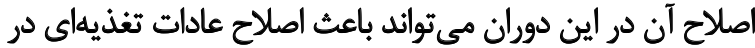

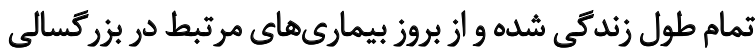

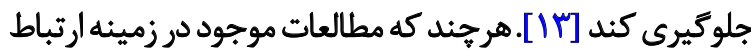

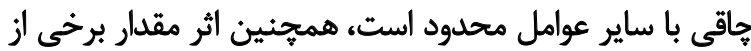

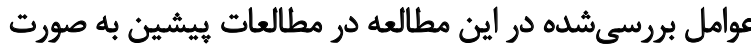

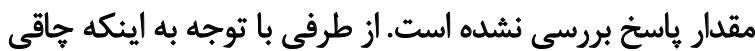

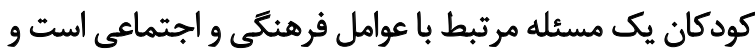

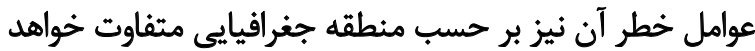

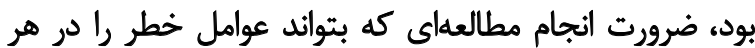
منطقه براى خاقى بررسى كند وجود دارئ مارد.

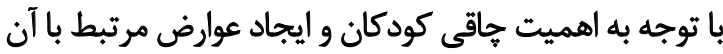

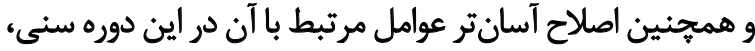

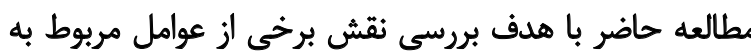

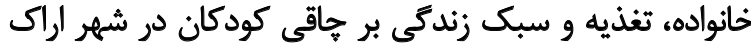

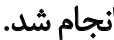

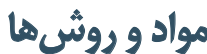

اين مطالعه مورد شاهدى همسانشده سنى است كه يس كإن

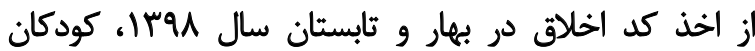

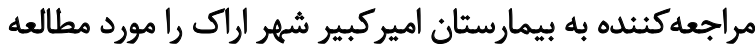

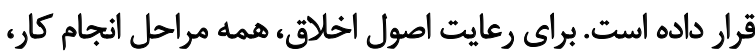

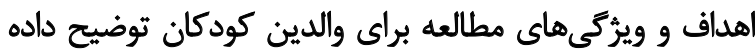

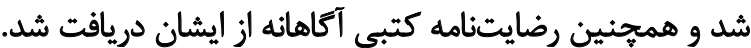

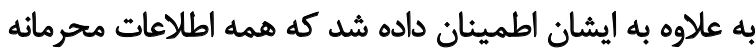

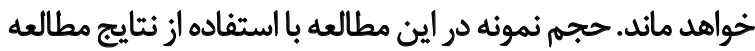

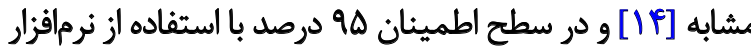

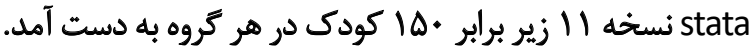

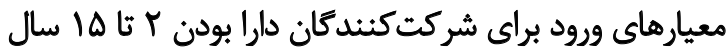

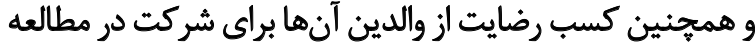
بود و معيارهاى خروج شامل مصرف داروهاى افزاينده يا كاهنده 
يك فرزند داشت نسبت شانس هاقي در كودكانى كه خانواده

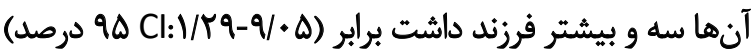

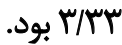

نتايج بررسى ارتباط بين هاقى و عوامل مربوط به تغذيه در

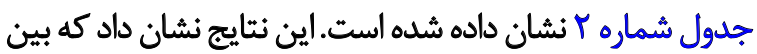

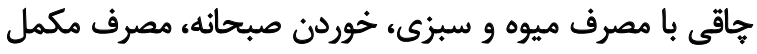

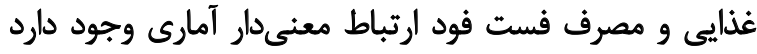

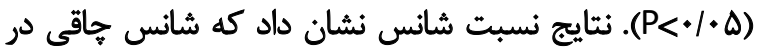

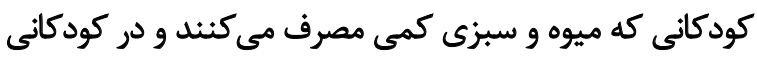

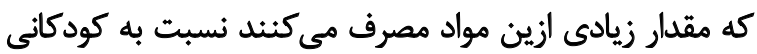

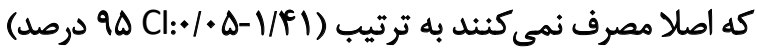

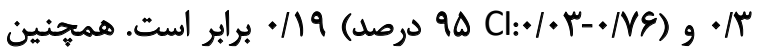

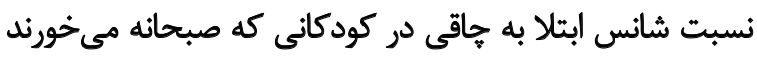

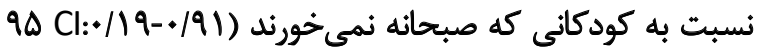

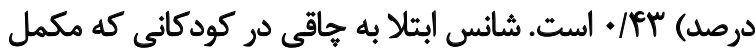

در جدول شماره ا نشان داده شده است. بر اساس اين نتايج

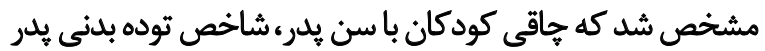

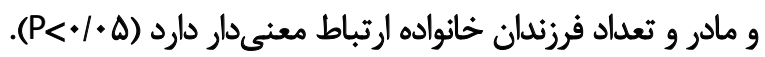

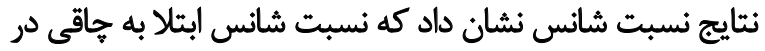

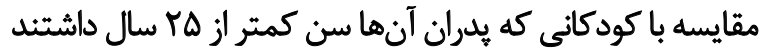

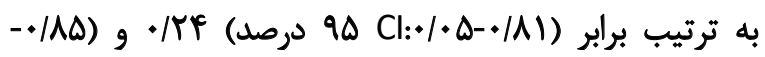

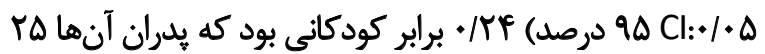

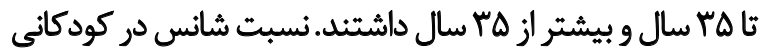

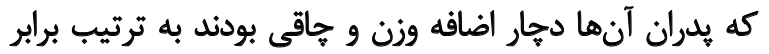

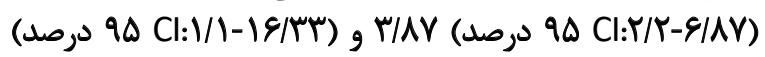

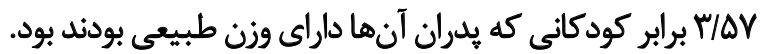

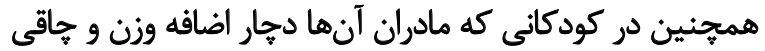

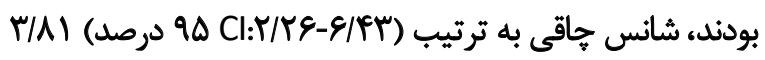

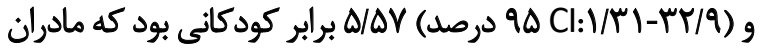

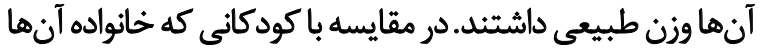

جدول ا. ارتباط بين هاقي و متغيرهاي مربوط به خانواده كودى

\begin{tabular}{|c|c|c|c|c|c|c|}
\hline \multirow{2}{*}{ حدود اطمينان } & \multirow{2}{*}{ نسبت شانس } & \multirow{2}{*}{ حد معنى دارى } & \multicolumn{2}{|c|}{ تعداد (درصد) } & \multirow{2}{*}{\multicolumn{2}{|c|}{ متغيرها }} \\
\hline & & & تروه بيمار & تروه كتترل & & \\
\hline \multirow{2}{*}{$\begin{array}{c}\text { رفرنس } \\
\text { رفV-I/TE }\end{array}$} & & \multirow{2}{*}{.$/ 491$} & $g\left(p^{e}\right)$ & $\operatorname{ar}(\lceil\wedge)$ & دختر & \multirow{2}{*}{ جنس } \\
\hline & $\cdot / N A$ & & $\operatorname{Ar}(\Delta \&)$ & $9 \pi(\& T)$ & ل بسر & \\
\hline \multirow{3}{*}{$\begin{array}{c}\text { رفرنس } \\
+\Delta-|A| \\
+\Delta-\mid A \Delta\end{array}$} & 1 & & $10(1 \cdot)$ & $F(T / V)$ & كمتر از ه广 سال & \multirow{3}{*}{ سن بلر } \\
\hline & $+/ T r$ & 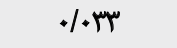 & A) (AH) & $M(\Delta N Y)$ & 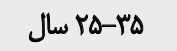 & \\
\hline & $\cdot / \pi r$ & & $\Delta f(r E)$ & $\triangle A(N N E)$ & بيشتر از هـ" سال & \\
\hline \multirow{3}{*}{$\begin{array}{c}\text { رفرنس } \\
. / 10-1 / Y A \\
. / 1 Y-1 / 19\end{array}$} & 1 & & $10(1)$. & $V(F / V)$ & كمتر از هT سال & \multirow{3}{*}{ سن مادر } \\
\hline &.$/ 10$ & . MAP & $99(89)$ & $1.1($ EV/T) & 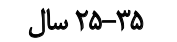 & \\
\hline &.$/ f$ & & $r E\left(M^{\circ}\right)$ & Pr (TA) & بيشتر از هب سال & \\
\hline \multirow{3}{*}{ 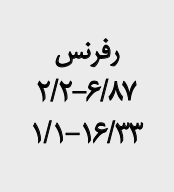 } & 1 & & $V \Delta(\Delta \cdot)$ & $119(\mathrm{Vq} / \mathrm{r})$ & طبيعى & \multirow{3}{*}{ شاخص توده بلنيى يلر } \\
\hline & r/AV & $\%$ & $\operatorname{se}(F+)$ & $r V(M)$ & اضافه وزن & \\
\hline & W/QV & & $9(9)$ & $P(T / M)$ & جاقى & \\
\hline \multirow{3}{*}{ 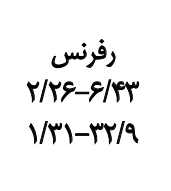 } & 1 & & $\Delta V(r \wedge)$ & $1.8(V+N)$ & طبيعى & \multirow{3}{*}{ شاخص توده بدنى } \\
\hline & $r / \Lambda 1$ & $\%$ & $\operatorname{Af}(\Delta \varepsilon)$ & HI (TV/M) & اضافه وزن & \\
\hline & $\Delta / \Delta V$ & & $9(9)$ & $r(r)$ & جاقّى & \\
\hline رفرنس & 1 & \multirow{3}{*}{$.1+.9$} & $9(9)$ & $M(1 F)$ & يكى & \multirow{3}{*}{ تعلداد فرزندان } \\
\hline$\cdot / M-\Delta / 9 q$ & $r / I V$ & & A) (AH) & $\operatorname{AV}(\Delta \mathrm{A})$ & دو & \\
\hline $1 / r q-q / \cdot \Delta$ & $r / m$ & & $\varepsilon \cdot(\varphi \cdot)$ & Pr (TA) & سله و بيشتر & \\
\hline
\end{tabular}

حد معنى دارى آزمونها با آزمون كاى مربع و همجنين نسبت شانس با ركرسيون لجستيك شرطى و با در نظر كرفتن خطاى هـ٪ محاسبه كرديده است. 
جدول ז. ارتباط بين جاقي و متغيرهاى مربوط به عوامل تغذيهاي

\begin{tabular}{|c|c|c|c|c|c|c|}
\hline \multirow{2}{*}{ حدود اطمينان } & \multirow{2}{*}{ نسبت شانس } & \multirow{2}{*}{ حد معني فارى } & \multicolumn{2}{|c|}{ تعداد (درصد) } & & \multirow{2}{*}{ هتغيرها } \\
\hline & & & ئروه ييمار & كروه كتترل & & \\
\hline رفرنس & 1 & \multirow{3}{*}{$.1 . . .1$} & $\mathbb{I r}(\Lambda)$ & $r(r)$ & اصلا & \multirow{3}{*}{ هصرف ميوه و سبزى } \\
\hline$.|+\Delta-| /|+|$ &.$/ \pi \varphi$ & & $\Delta 1(\pi)$ & MV (YPIV) & كم & \\
\hline$+/ . r-+N E$ & .119 & & $\operatorname{AV}(\Delta A)$ & $M \cdot(N / r)$ & زياد & \\
\hline رفرنس & 1 & \multirow{2}{*}{$.1 . .1$} & $M(M)$ & $\mathbb{I r}(\mathrm{NV})$ & خير & \multirow{2}{*}{ خوردن صبحانه } \\
\hline$+/ 19-+/ 91$ & . & & $\operatorname{Irr}(A T)$ & $\operatorname{Irv}(q) / \pi)$ & بله & \\
\hline رفرنس & 1 & \multirow{2}{*}{$+1+\cdots+1$} & $\operatorname{Af}(\Delta \&)$ & $\| \varepsilon(W / N)$ & خير & \multirow{2}{*}{ هصرف مكمل غذايى } \\
\hline$V / \Delta A-F / \Delta V$ & T/EA & & $g 8(P t)$ & $M F(T r / V)$ & بله & \\
\hline رفرنس & 1 & \multirow{4}{*}{.$|\cdot \Delta|$} & $F(T / V)$ & $9(8)$ & نمىكند & \multirow{4}{*}{ مصرف ميان وعده } \\
\hline ./1A-YQ/9 & F/AY & & $\operatorname{Ir}(N V)$ & $q\left(f^{\infty}\right)$ & يك بار & \\
\hline$.|8 q-| r \mid \cdot 8$ & r/at & & $91(8 \cdot / 8)$ & $M(\Delta Y)$ & دوبار & \\
\hline . /PT-V/AT & V/Fa & & $\operatorname{Pr}(\mathrm{YA})$ & $\Delta V(N A)$ & سه بار و ييشتر & \\
\hline رفرنس & 1 & \multirow{2}{*}{.1 .85} & $\Delta V($ FN) & $\left.n^{N}(\Delta) / \pi\right)$ & خير & \multirow{2}{*}{ هصرف غناي خارج خاثه } \\
\hline$. / Q \Delta-Y(\Delta)$ & $V / \Delta F$ & & $9 \pi\left(\mathscr{F}^{+}\right)$ & $W(F N V)$ & بله & \\
\hline رفرنس & 1 & \multirow{2}{*}{$+1+\ldots+1$} & $91(\xi \cdot / V)$ & $\operatorname{lrq}(N)$ & خير & \multirow{2}{*}{ مصرف فست فود } \\
\hline$r / 19-V / T r$ & $r / u$ & & $\Delta Q(r q / \%)$ & $M(M P)$ & بله & \\
\hline
\end{tabular}

حد معنى دارى آزمونها با آزمون كاى مربع و همجنين نسبت شانس با ركرسيون لجستيك شرطى و با در نظر كرفتن خطاى هـ/ محاسبه شده است.

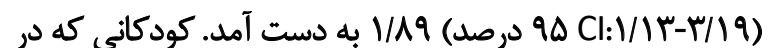

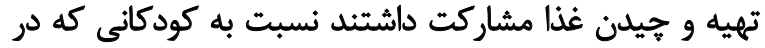

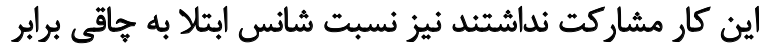

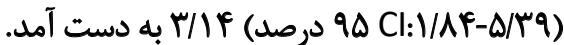

\section{بحث}

بر اساس نتايج مطالعه حاضر برخى از عوامل مربوط به خانواده

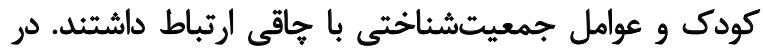

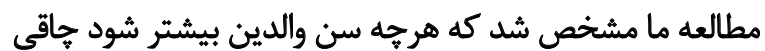

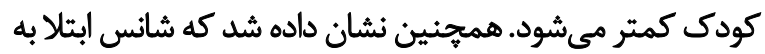

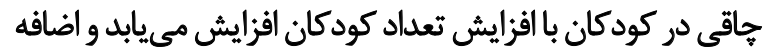

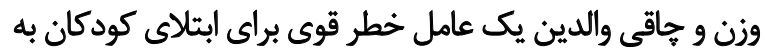

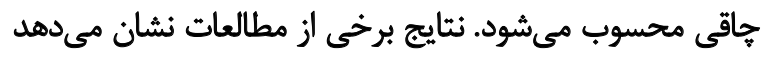

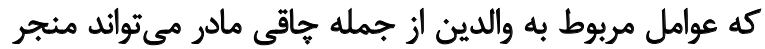

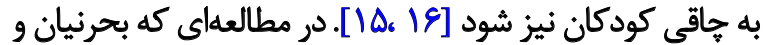

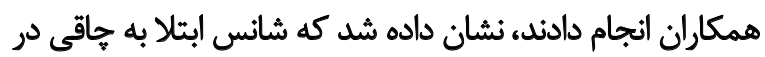

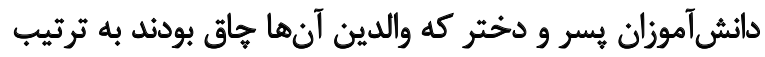

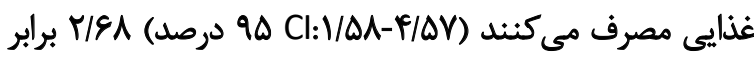

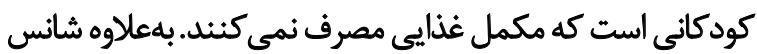

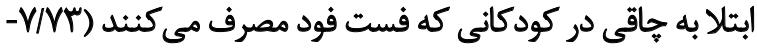

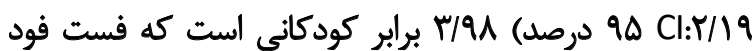

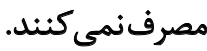

نتايج بررسى ارتباط بين جاقى و عوامل مربوط به سبك زندكى إنى

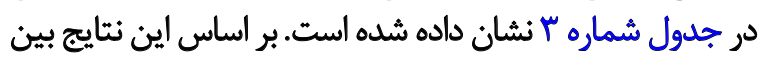

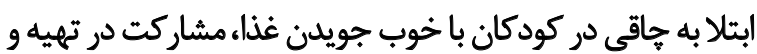

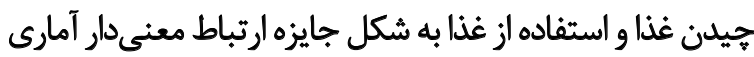

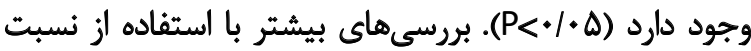

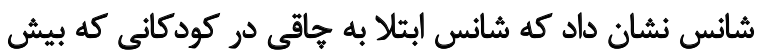

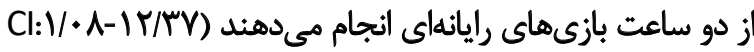

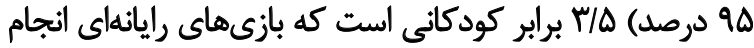

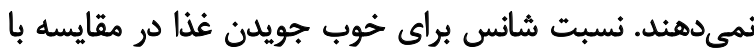

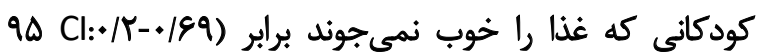

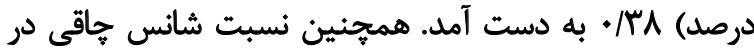
كودكانى كه از غذا به شكل جايزه براي آنه هـا استفاده مى نشد برابر 
جدول ץ. ارتباط بين جاقى و متغيرهاى مربوط به عوامل سبك زندكى

\begin{tabular}{|c|c|c|c|c|c|c|}
\hline \multirow{2}{*}{ اطمينان } & \multirow{2}{*}{ نسبت شانس } & \multirow{2}{*}{ حد معنى دارى } & \multicolumn{2}{|c|}{ تعداد (درصد) } & & \multirow{2}{*}{ متغيرها } \\
\hline & & & ئووه بيمار & تروه كثترل & & \\
\hline رفرنس & 1 & \multirow{2}{*}{$\cdot / r \cdot r$} & $111\left(M^{*}\right)$ & $1 . r(E N V)$ & & \multirow{2}{*}{ فعاليت ورزشيى } \\
\hline$. / P Q-\mid / M$ &.$/ 18$ & & rq (TE) & $\operatorname{er}\left(r^{\prime} / r^{\prime}\right)$ & دارد & \\
\hline رفرنس & 1 & \multirow{4}{*}{$+1+11$} & $\operatorname{me}(18)$ & YA (INV) & خير & \multirow{4}{*}{ انجام بازيهاى رايانهاى } \\
\hline$. / \Delta H^{-}-r / \Delta r$ & $1 / 18$ & & $r q(\pi)$ & $\Psi(M P)$ & كمتر از اساعت & \\
\hline$\cdot / \Delta Y-Y / \cdot V$ & $1 / \cdot \Delta$ & & $r(\varphi A)$ & $\Lambda \cdot(\Delta r / r)$ & r- (- ساعت & \\
\hline$M \cdot A-I R / H Z$ & $m / \Delta$ & & $M(M)$ & $q(\varphi)$ & بيشتر از r ساعت & \\
\hline رفرنس & 1 & \multirow[t]{2}{*}{.1 .98} & $N(\Delta N N)$ & $M(\Delta N V)$ & كمتر از 9 ساعت & \multirow{2}{*}{ زمان خوابيلن } \\
\hline$/ q 4-r / 4 q$ & V/Ar & & $V A(\Delta r)$ & $\operatorname{sr}(r \mid / M)$ & بيشتر از و ساعت & \\
\hline رفرنس & 1 & \multirow{2}{*}{ - rqu } & $\operatorname{Ar}(\Gamma \Delta / T)$ & 9) $(\varepsilon+/ V)$ & خير & \multirow{2}{*}{ وادار كردن به غذا خوردن } \\
\hline$\cdot / R A-r / \cdot V$ & $I / T Y$ & & $\operatorname{~} A \Lambda(\Delta F / V)$ & $\Delta q(r q / r)$ & بله & \\
\hline رفرنس & 1 & \multirow{2}{*}{.$|8 A|$} & If $\left(\mathscr{q} / T^{2}\right)$ & $\pi(A)$ & خير & \multirow{2}{*}{ غذا خوردن خانواده با هم } \\
\hline$\cdot m e-r / \cdot P$ & ./AF & & $\operatorname{lrg}(9 . M)$ & IHA (9Y) & بله & \\
\hline & 1 & \multirow[t]{2}{*}{$+\infty+\infty$} & PA (MT) & $\pi(1 \Delta / r)$ & خير & \multirow{2}{*}{ خوب جويلن غذا } \\
\hline$\cdot \pi \cdot-\cdot 189$ & . / & & I.Y (8A) & $\operatorname{Irr}(A+M)$ & بله & \\
\hline رفرنس & 1 & \multirow{2}{*}{$.1 \%$} & rq (Te) & $8 \cdot\left(t^{*}\right)$ & خير & \multirow{2}{*}{ استفاده از غذا به عثوان جايزه } \\
\hline$V / 1 r-r / 19$ & $1 / 19$ & & $m(1)$ & $q+(\varepsilon+)$ & بله & \\
\hline رفرنس & 1 & \multirow{2}{*}{$.1 . . .1$} & A) (Af) & $114(N N V)$ & خير & \multirow{2}{*}{ مشاركت كودى در تهيه و } \\
\hline V/AF-ه/Fq & $r / / F$ & & $89(48)$ & $M(r M / M)$ & 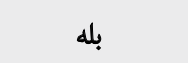 & \\
\hline رفرنس & 1 & \multirow{3}{*}{./RAY } & $M(1 F)$ & $I f(q / \pi)$ & كمتر از ب ليوان & \multirow{3}{*}{ مصرف آب در طول روز } \\
\hline$. / 4 A-1 / 49$ & .194 & & $\operatorname{Ir\Delta }(\Delta T / T)$ & $M+(N E N)$ & F- ب ليوان & \\
\hline$\cdot \mid \cdot V-r / M$ &.$/ A F$ & & $F(T / V)$ & $\varepsilon(\varphi)$ & بيشتر از † ليوان & \\
\hline
\end{tabular}

حد معنى دارى آزمونها با آزمون كاى مربع و همجنين نسبت شانس با ركرسيون لجستيك شرطى و با در نظر كرفتن خطاى هـ / محاسبه شده است.

است بر هاقي كودك تاثير دارد. نتايج مطالعات مختلف نشان داده

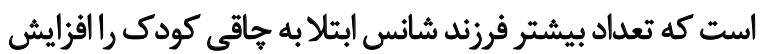

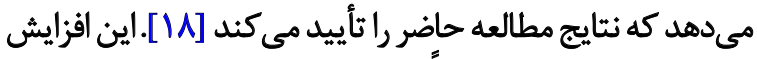

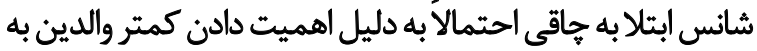

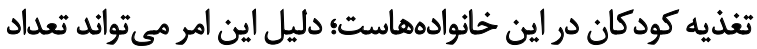

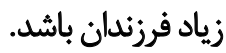

يكى ديغر از عوامل جمعيتشناختى بررسىشده در مطالعه حاضر سن مادر و جنسيت كودك بود كه اثر اين دو متغير بردي

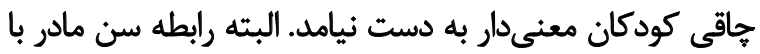

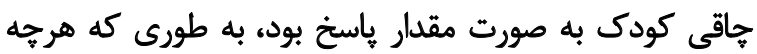

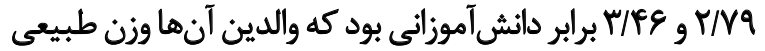

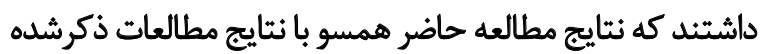

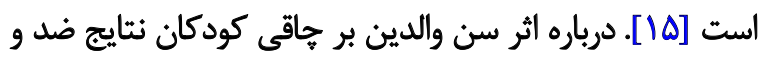
نقيضى وجود دارد. برخى از مطالعات بيان كردهاند كه كود كانى إنى

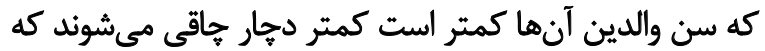

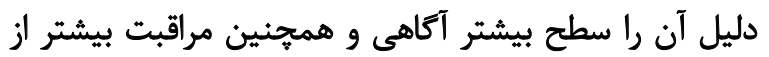

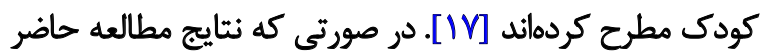

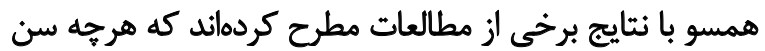

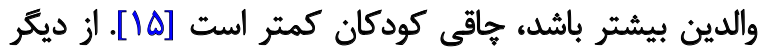

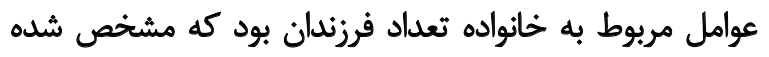


مىشود. كوزبيكا و همكاران در مطالعهاي ارتباط شاخص توده

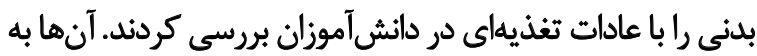

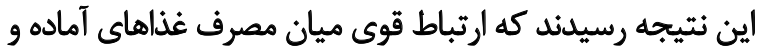

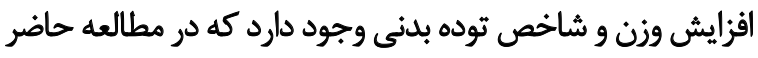

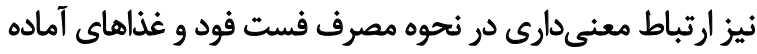

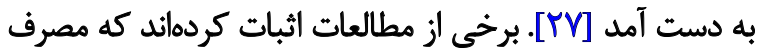

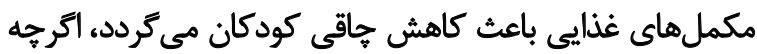

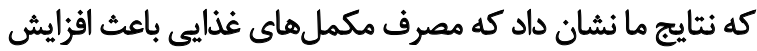

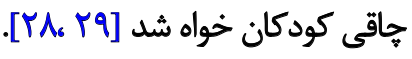

يكى ديكر از عادات غُذايى كودكان جاق مصرف ميان وعدههاى

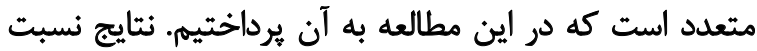

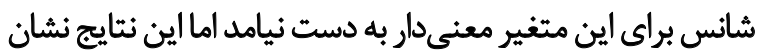

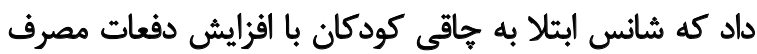

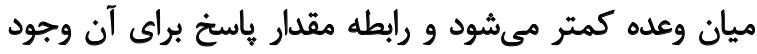

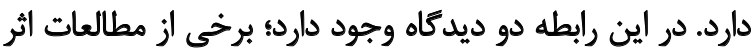

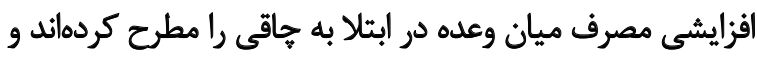

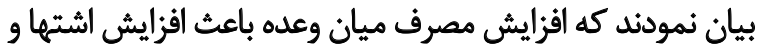

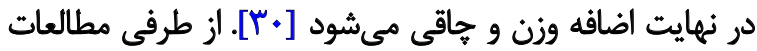

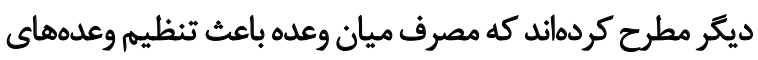

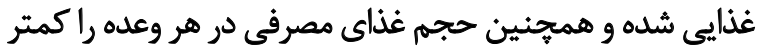

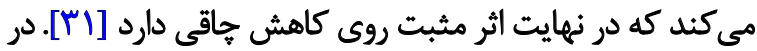

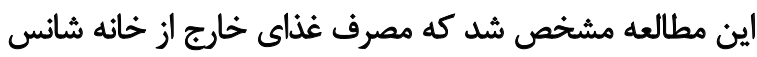

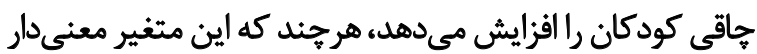

بر اساس نتايج مطالعه حاضر برخى از عوامل مربوط به سبك

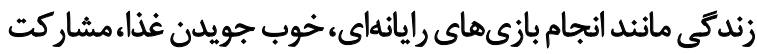

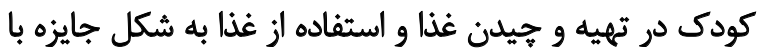

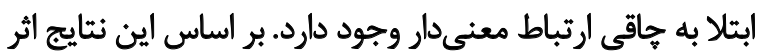

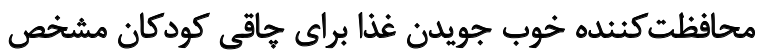

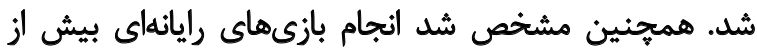

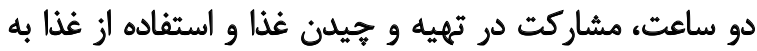

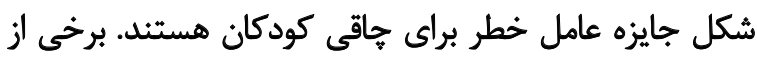

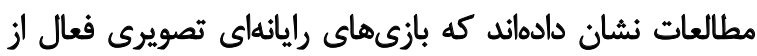

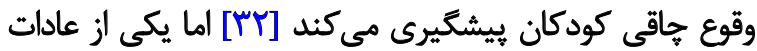

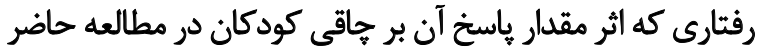

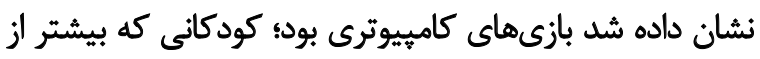

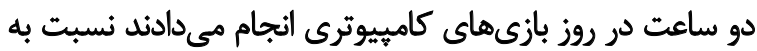

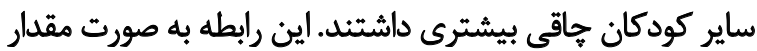

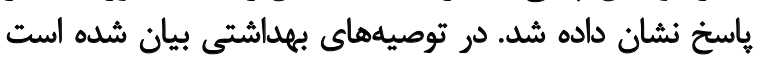

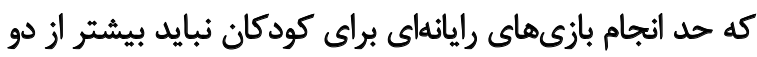

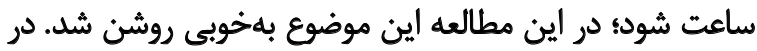

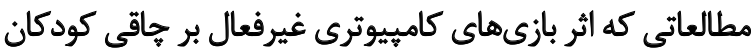

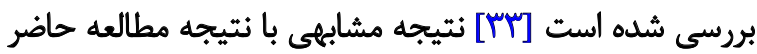

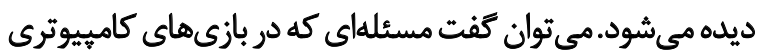

سن مادر بيشتر بود شانس ابتلا به جاقى كودى كمتر مي شئد

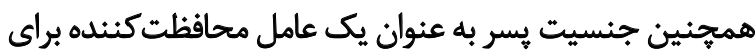

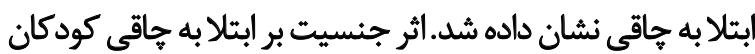

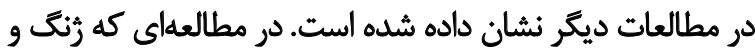

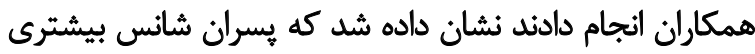

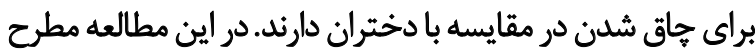

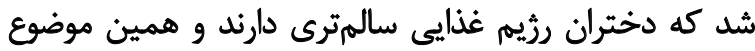

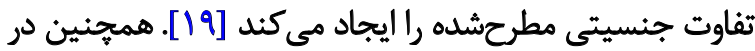

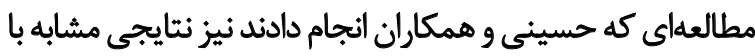

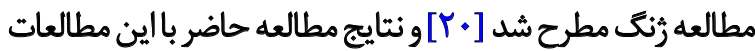

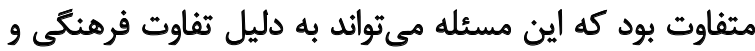
جغرافيايى جمعيت مطالعهشده حاضر با ساير مطالعات باشند.

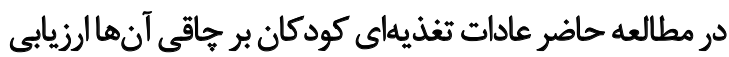

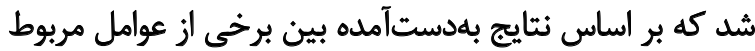

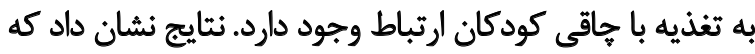

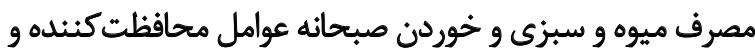

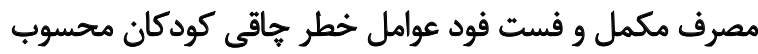

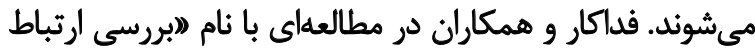

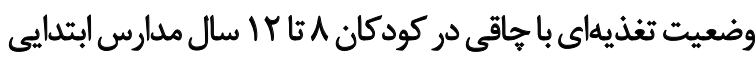

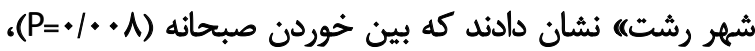

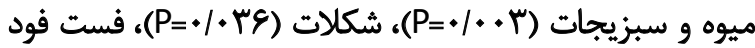

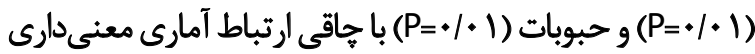

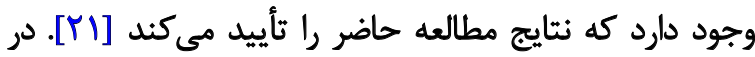

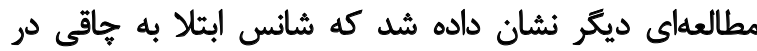

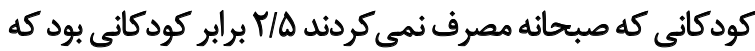

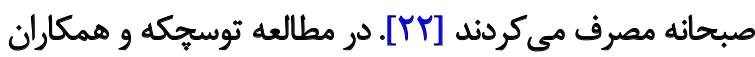

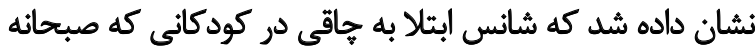

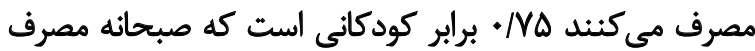

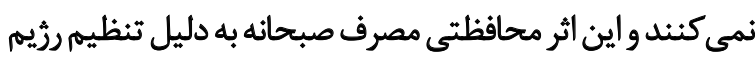

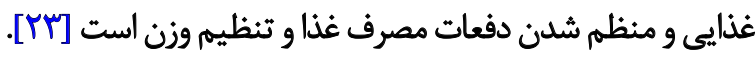
در مطالعه دوآن و همكاران نشان داده شد كه بعضى از رفتارهاى

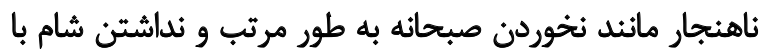

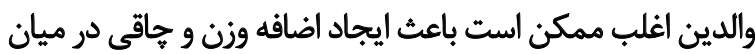

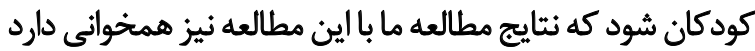

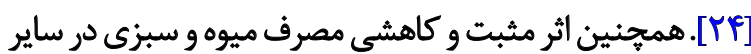

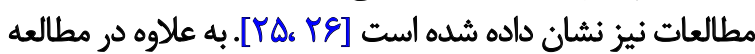

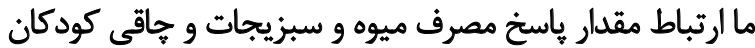

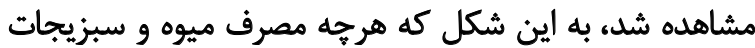

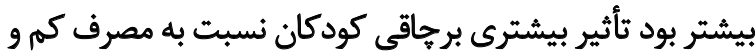

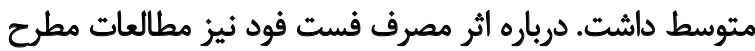

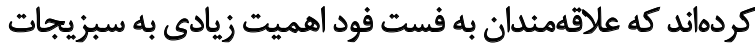

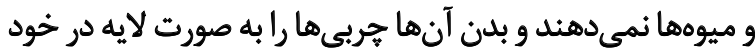

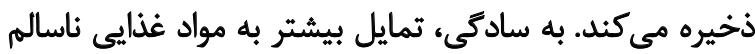

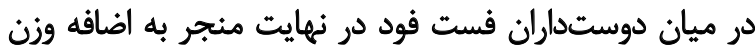




\section{نتيجلكَيرى}

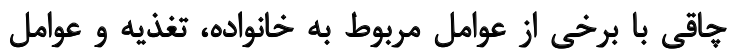

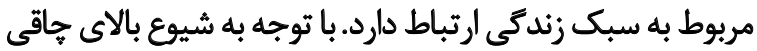

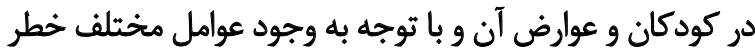

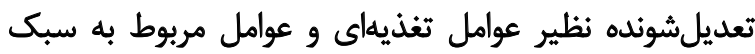

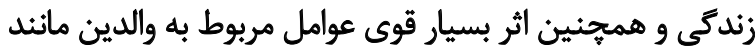

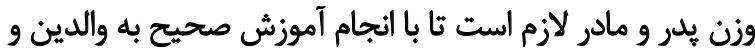

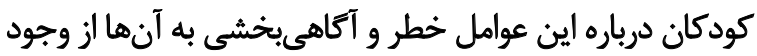

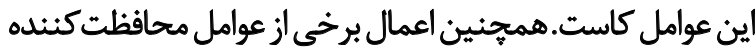

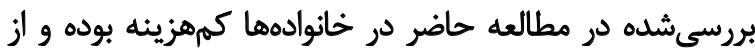

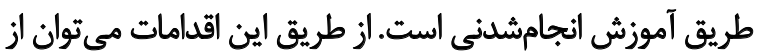
ميزان ابتلابه جاقى كودكان كاست.

ملاحظات اخلاقي

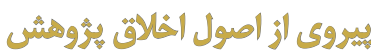

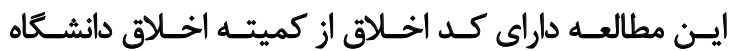

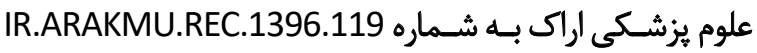
سمث

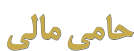

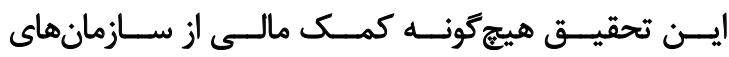

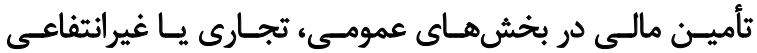
دريافتـ نكـرد.

on

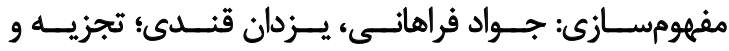

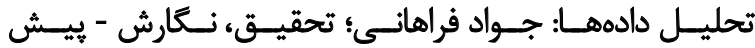

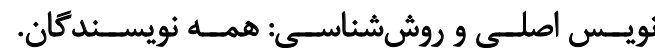

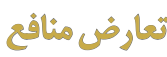

نويسندًان مقاله هيجَّّنه تعارضى در منافع اعلام نكردند.

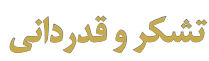

بدينوسيله از كليه يرسئل بيمارستان اميركبير شهر اراى

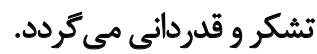

باعث جاقى كودكان مىشود، كمتحركى است، زيرا در مطالعاتى

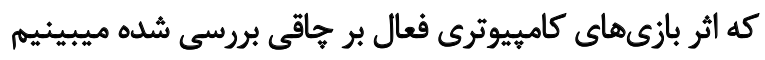

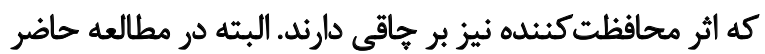

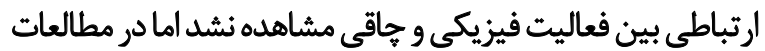

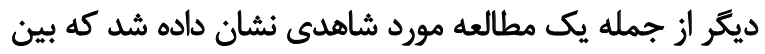

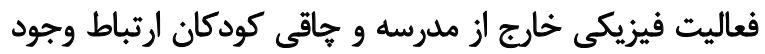

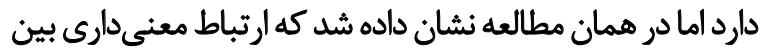

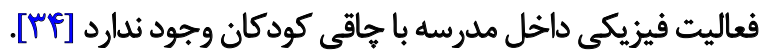

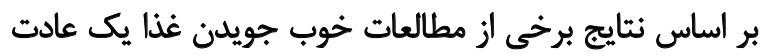

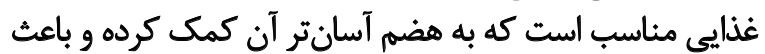

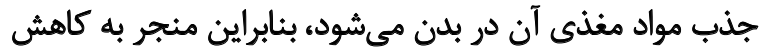

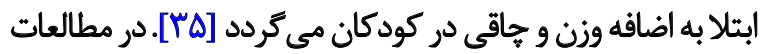

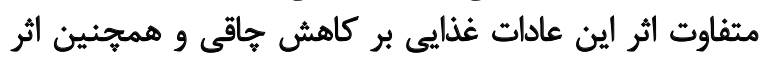

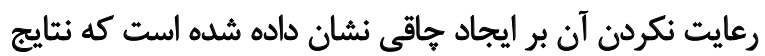

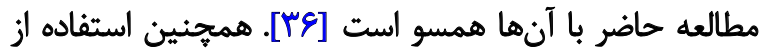

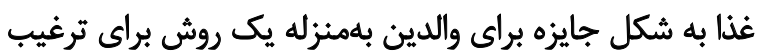

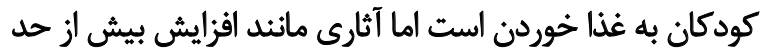

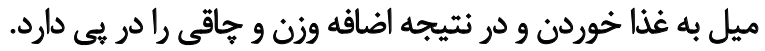

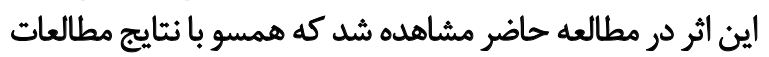

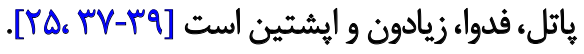

در مطالعه حاضر رابطه بين زمان خوابيدن، وادار كردن كودى

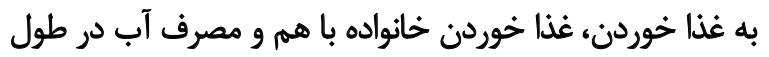

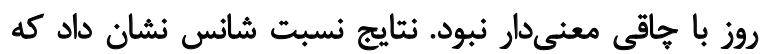

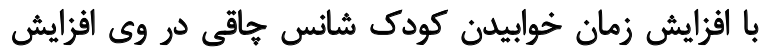

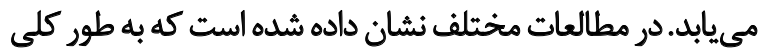

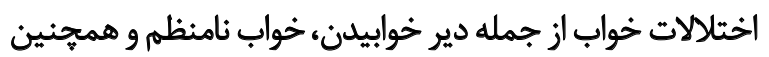

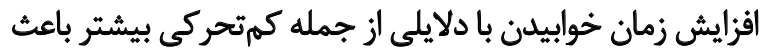

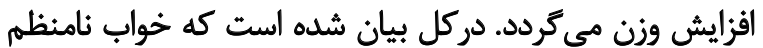

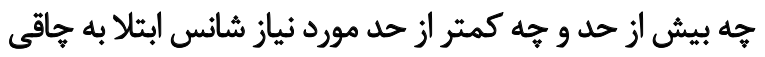

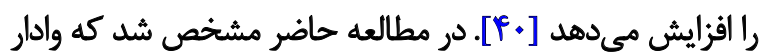

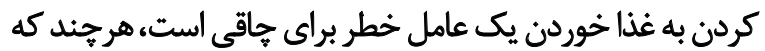

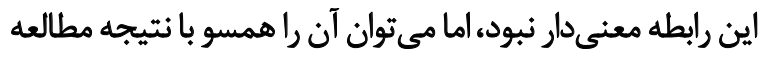

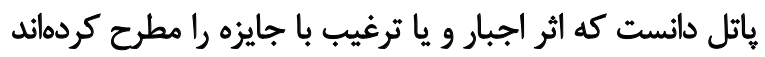

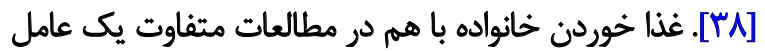

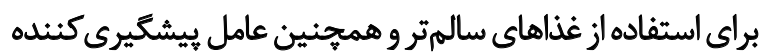

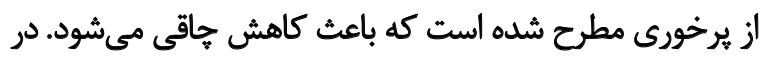

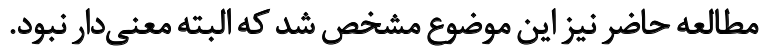

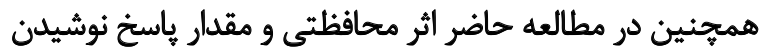

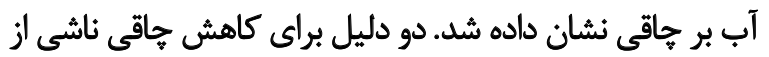

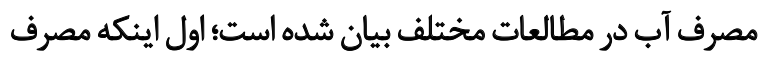

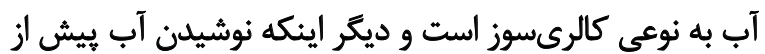

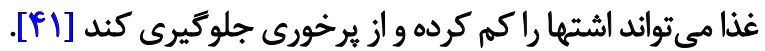




\section{Refrences}

[1] Santas F, Santas G. Prevalence of pre-school children for overweight/ obesity in Turkey. World J Pediatr. 2018; 14(1):77-83. [DOI:10.1007/ s12519-017-0103-9][PMID]

[2] Kearns K, Dee A, Fitzgerald AP, Doherty E, Perry IJ. Chronic disease burden associated with overweight and obesity in Ireland: The effects of a small BMI reduction at population level. BMC Public Health. 2014; 14:143. [DOI:10.1186/1471-2458-14-143]

[3] Nadeau KJ, Maahs DM, Daniels SR, Eckel RH. Childhood obesity and cardiovascular disease: Links and prevention strategies. Nat Rev Cardiol. 2011; 8(9):513-25. [DOI:10.1038/nrcardio.2011.86] [PMID] [PMCID]

[4] Weiss R, Kaufman FR. Metabolic complications of childhood obesity: Identifying and mitigating the risk. Diabetes Care. 2008; 31(Suppl 2):S310-6. [DOI:10.2337/dc08-s273] [PMID]

[5] Sahoo K, Sahoo B, Choudhury AK, Sofi NY, Kumar R, Bhadoria AS. Childhood obesity: Causes and consequences. J Family Med Prim Care. 2015; 4(2):187-92. [DOI:10.4103/2249-4863.154628] [PMID] [PMCID]

[6] Shivpuri A, Shivpuri A, Sharma S. Childhood obesity: Review of a growing Problem. Int J Clin Pediatr Dent. 2012; 5(3):237-41. [DOI:10.5005/jp-journals-10005-1175] [PMID] [PMCID]

[7] Djalalinia S, Kelishadi R, Qorbani M, Peykari N, Kasaeian A, Nasli-Esfahani $E$, et al. A systematic review on the prevalence of overweight and obesity, in Iranian children and adolescents. Iran J Pediatr. 2016; 26(3):e2599. [DOI:10.5812/ijp.2599] [PMID] [PMCID]

[8] Walls HL, Peeters A, Son PT, Quang NN, Hoai NTT, Viet NL, et al. Prevalence of underweight, overweight and obesity in urban Hanoi, Vietnam. Asia Pac J Clin Nutr. 2009; 18(2):234-9. [PMID]

[9] Fonseca H, Silva A, Matos M, Esteves I, Costa P, Guerra A, et al. Validity of BMI based on self-reported weight and height in adolescents. Acta Pediatr. 2010; 99(1):83-8. [DOI:10.1111/j.16512227.2009.01518.x] [PMID]

[10] Papandreou D, Makedou K, Zormpa A, Karampola M, Ioannou A, Hitoglou-Makedou A. Are dietary intakes related to obesity in children? Open Access Maced J Med Sci. 2016; 4(2):194-9. [DOI:10.3889/oamjms.2016.045] [PMID] [PMCID]

[11] Braithwaite I, Stewart AW, Hancox RJ, Beasley R, Murphy R, Mitchell EA. Fast-food consumption and body mass index in children and adolescents: An international cross-sectional study. BMJ Open. 2014; 4(12):e005813. [DOI:10.1136/bmjopen-2014-005813][PMID] [PMCID]

[12] Irazusta A, Hoyos I, Irazusta J, Ruiz F, Díaz E, Gil J. Increased cardiovascular risk associated with poor nutritional habits in first-year university students. Nutr Res. 2007; 27(7):387-94. [DOI:10.1016/j. nutres.2007.05.007]

[13] Drewnowski A. Obesity, diets, and social inequalities. Nutr Rev. 2009; 67(Suppl 1):S36-9. [DOI:10.1111/j.1753-4887.2009.00157.x] [PMID]

[14] Shakeri M, Mojtahedi Y, Moradkhani M. [Obesity among female adolescents of Tehran schools (Persian)]. J Payavard Salamat. 2013; 6(5):403-11. https://payavard.tums.ac.ir/browse.php?a_ id $=16 \&$ sid $=1 \&$ slc_lang=en

[15] Bahreynian M, Qorbani M, Khaniabadi BM, Motlagh ME, Safari O, Asayesh $\mathrm{H}$, et al. Association between obesity and parental weight status in children and adolescents. J Clin Res Pediatr Endocrinol. 2017; 9(2):111-7. [DOI:10.4274/jcrpe.3790] [PMID] [PMCID]
[16] Fuemmeler BF, Lovelady CA, Zucker NL, Ostbye T. Parental obesity moderates the relationship between childhood appetitive traits and weight. Obesity (Silver Spring). 2013; 21(4):815-23. [DOI:10.1002/oby.20144] [PMID] [PMCID]

[17] Khashayar P, Kasaeian A, Heshmat R, Motlagh ME, Mahdavi Gorab A, Noroozi $M$, et al. Childhood overweight and obesity and associated factors in Iranian children and adolescents: A multilevel analysis: The CASPIAN-IV study. Front Pediatr. 2018; 6:393. [DOI:10.3389/ fped.2018.00393] [PMID] [PMCID]

[18] Karki A, Shrestha A, Subedi N. Prevalence and associated factors of childhood overweight/obesity among primary school children in urban Nepal. BMC Public Health. 2019; 19(1):1055. [DOI:10.1186/ s12889-019-7406-9] [PMID] [PMCID]

[19] Zhang P, Wu H, Zhou X, Lu Y, Yuan Z, Moore JB, et al. The association between family and parental factors and obesity among children in Nanchang, China. Front Public Health. 2016; 4:162. [DOI:10.3389/ fpubh.2016.00162] [PMID] [PMCID]

[20] Hosseini SM, Latifi S, Kelishadi R. Analysis of factors affecting the body mass index in a national sample of Iranian children and adolescents: Bootstrapping regression. Adv Biomed Res. 2017; 6:152 [DOI:10.4103/2277-9175.219417] [PMID] [PMCID]

[21] Fadakar R, Shokrzadeh P, Pasha A, KazemNezhad Leili E. [The relationship between nutritional status and obesity in 8-12 years old children of Rasht city (Persian)]. J Health Care. 2018; 19(4):212-20. http://hcjournal.arums.ac.ir/article-1-895-en.html

[22] Champilomati G, Notara V, Prapas C, Konstantinou E, Kordoni M Velentza $A$, et al. Breakfast consumption and obesity among preadolescents: An epidemiological study. Pediatr Int. 2020; 62(1):81-8 [DOI:10.1111/ped.14050] [PMID]

[23] Toschke AM, Thorsteinsdottir KH, von Kries R, GME Study Group. Meal frequency, breakfast consumption and childhood obesity. Int J Pediatr Obes. 2009; 4(4):242-8. [DOI:10.3109/17477160902763341] [PMID]

[24] Duan R, Xue H, Liu Y, Yang M, Cheng G. [Cross-sectional association between eating behaviors and overweight/obesity among children and adolescents in Chengdu city (Chinese)]. Wei Sheng Yan Jiu. 2015; 44(4):576-80. [PMID]

[25] Epstein LH, Gordy CC, Raynor HA, Beddome M, Kilanowski CK Paluch R. Increasing fruit and vegetable intake and decreasing fat and sugar intake in families at risk for childhood obesity. Obes Res. 2001 9(3):171-8. [DOI:10.1038/oby.2001.18] [PMID]

[26] Korinek EV, Bartholomew JB, Jowers EM, Latimer LA. Fruit and vegetable exposure in children is linked to the selection of a wider variety of healthy foods at school. Matern Child Nutr. 2015; 11(4):999-1010. [DOI:10.1111/mcn.12035] [PMID] [PMCID]

[27] Kuźbicka K, Rachoń D. Bad eating habits as the main cause of obesity among children. Pediatr Endocrinol Diabetes Metab. 2013 19(3):106-10. [PMID]

[28] Almuhanna MA, Alsaif M, Alsaadi M, Almajwal A. Fast food intake and prevalence of obesity in school children in Riyadh city. Sudan $J$ Paediatr. 2014; 14(1):71-80. [PMID] [PMCID]

[29] Zhao $Y$, Wang L, Xue H, Wang $H$, Wang $Y$. Fast food consumption and its associations with obesity and hypertension among children: Results from the baseline data of the Childhood Obesity Study in China Mega-cities. BMC Public Health. 2017; 17(1):933. [DOI:10.1186/ s12889-017-4952-x] [PMID] [PMCID] 
[30] Rudy E, Bauer KW, Hughes SO, O'Connor TM, Vollrath K, Davey A, et al. Interrelationships of child appetite, weight and snacking among Hispanic preschoolers. Pediatr Obes. 2018; 13(1):38-45. [DOI:10.1111/ ijpo.12186] [PMID] [PMCID]

[31] Roblin L. Childhood obesity: Food, nutrient, and eating-habit trends and influences. Appl Physiol Nutr Metab. 2007; 32(4):635-45. [DOI:10.1139/H07-046][PMID]

[32] Lu AS, Kharrazi H, Gharghabi F, Thompson D. A systematic review of health videogames on childhood obesity prevention and intervention. Games Health J. 2013; 2(3):131-41. [DOI:10.1089/g4h.2013.0025] [PMID][PMCID]

[33] LeBlanc AG, Chaput JP, McFarlane A, Colley RC, Thivel D, Biddle SJ, et al. Active video games and health indicators in children and youth: $A$ systematic review. PloS One. 2013; 8(6):e65351. [DOI:10.1371/journal. pone.0065351] [PMID][PMCID]

[34] Bhuiyan MU, Zaman S, Ahmed T. Risk factors associated with overweight and obesity among urban school children and adolescents in Bangladesh: A case-control study. BMC Pediatr. 2013; 13:72. [DOI:10.1186/1471-2431-13-72] [PMID][PMCID]

[35] Slim R, Honein K, Yaghi C, Chemaly M, Kheir B, Sayegh R. Chewing is an important first step in digestion. Diagnosis: Piece of glass in stomach. Gut. 2006; 55(3):379-424. [DOI:10.1136/gut.2005.075598] [PMID] [PMCID]

[36] Okubo H, Murakami K., Masayasu S, Sasaki S. The relationship of eating rate and degree of chewing to body weight status among preschool children in Japan: A nationwide cross-sectional study. Nutrients. 2018; 11(1):64. [DOI:10.3390/nu11010064] [PMID][PMCID]

[37] Fedewa AL, Davis MC. How food as a reward is detrimental to children's health, learning, and behavior. J Sch Health. 2015; 85(9):648-58. [DOI:10.1111/josh.12294] [PMID]

[38] Patel C, Karasouli E, Shuttlewood E, Meyer C. Food parenting practices among parents with overweight and obesity: A systematic review. Nutrients. 2018; 10(12):1966. [DOI:10.3390/nu10121966] [PMID][PMCID]

[39] Ziauddeen H, Alonso-Alonso M, Hill JO, Kelley M, Khan NA. Obesity and the neurocognitive basis of food reward and the control of intake. Adv Nutr. 2015; 6(4):474-86. [DOI:10.3945/an.115.008268] [PMID][PMCID]

[40] Miller AL, Lumeng JC, LeBourgeois MK. Sleep patterns and obesity in childhood. Curr Opin Endocrinol Diabetes Obes. 2015; 22(1):41-7. [DOI:10.1097/MED.0000000000000125] [PMID][PMCID]

[41] Schwartz AE, Leardo M, Aneja S, Elbel B. Effect of a school-based water intervention on child body mass index and obesity. JAMA Pediatr. 2016; 170(3):220-6. [DOI:10.1001/jamapediatrics.2015.3778] [PMID][PMCID] 